\title{
Adjust of energy with compactly supported orthogonal wavelet for steganographic algorithms using the scaling function $1 / \sqrt{2^{j}}$
}

\author{
Blanca E. Carvajal-Gámez*, Francisco J. Gallegos-Funes, Alberto J. Rosales-Silva and \\ José L. López-Bonilla
}

National Polytechnic Institute, Professional Unit Engineering and Advanced Technologies, Av. IPN 2580, Electronica, Barrio La Laguna Ticoman, 07740, Mexico D. F., Mexico.

Accepted 23 January, 2013

\begin{abstract}
When RGB images are processed for the implementation of steganographic algorithms, it is important to study the quality of the cover and retrieved images, since it typically used digital filters, reaching visibly deformed images. The discrete wavelet transform is useful for adjusting the stego- image quality, because one of their principal qualities which are able to analyze different frequency levels (multi-resolution analysis). By decomposing the image using the discrete wavelet transform, we obtain sub-images representing different levels of frequencies, in each of these sub-bands can expand or contract the obtained adjusting sub image well as possible to a desired quality. There by avoiding one of the main problems of steganography: distortion of stego-image. When a steganographic algorithm is employed, numerical calculations performed by the computer cause errors and alterations in the processed images, so we apply a scaling factor depending on the number of bits of the image to adjust these errors.
\end{abstract}

Key words: Steganographic algorithms, RGB images, scaling factor, wavelet.

\section{INTRODUCTION}

A classification of information hiding techniques is described in Petticolas and Katzenbeisser (2000). According to this classification, the information hiding techniques include convert communication, steganography, and digital watermarking. Steganography is the science that involves the secret communications of data in an appropriate multimedia carrier, such as, audio, image, and video files (Cheddad et al., 2010). It comes under the assumption that if the secret data is visible, the point of attack is evident (Cheddad et al., 2010; Petticolas and Katzenbeisser, 2000), thus, the goal here is to conceal the existence of the embedded data. In the case of image files, a steganographic method employs innocent-looking media called host or cover image to imperceptibly carry hidden data to an intended recipient (Cheddad et al., 2010; Petitcolas et al., 1999; Petticolas and Katzenbeisser, 2000). The camouflage of secret data

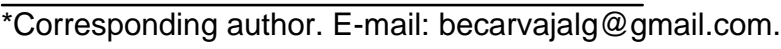

in stego-images to pass sensitive data between two parties can be regarded as secret communication, but it is different from cryptography. Cryptography encrypts the content of secret data into cipher texts and transmits the data in cipher to a receiver. However, using cryptography techniques merely for secret communication may fail since a cipher text is in meaningless form and thus, easily arouses the curiosity of malicious people who desire to recover or destroy data. Unlike cryptography, steganography conceals the fact that there is secret communication going on. A stego-image has meaning and the hidden data are camouflaged very well so that no one suspects that embedded data exist (Yuan et al., 2007).

For assured security, secret data used in steganography are usually encrypted using cryptography techniques before they are embedded into a host image. It is well known that the main concerns with steganographic methods are the hiding capacity $(\mathrm{HC})$ of the host image and the quality of the stego-image. It is different from the technique of watermarking. The 


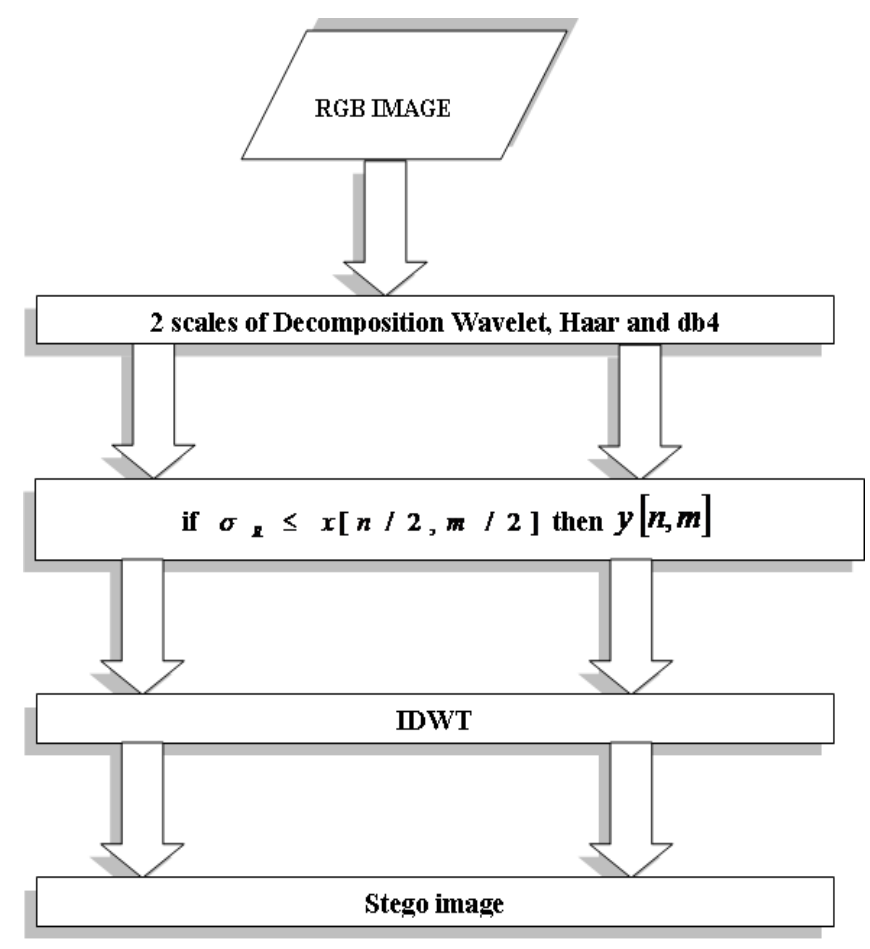

Figure 1. Block diagram of the steganographic algorithm.

purpose of watermarking is to protect the copyright of an image. The image embedded with the hidden data (that is, secret data, copyright notice, serial number) is called the stego-image and it looks as a normal image. Unintended recipients of a stego-image are unaware of the existence of the hidden data. A stego-key is used to control the hiding process so as to restrict detection and/or recovery of the embedded data (Petitcolas et al., 1999). A steganographic technique is usually evaluated in terms of the visual quality and the embedding capacity; in other words, an ideal steganographic scheme should have a large embedding capacity and excellent stegoobject visual quality. Unfortunately, the fact is that the visual quality degradation is in proportion to the embedding capacity, and to push one to the limit really means to totally sacrifice the other. The more reasonable way to deal with this trade-off situation is probably to strike a balance between the two (Wu and Hwang, 2007). Judging by whether the human vision sensitivity is considered in the design of the embedding algorithm, we can categorize the schemes into three types: (1) high embedding capacity schemes with acceptable image quality (Chan and Cheng, 2004; Lin and Tsai, 2004; Thien and Lin, 2003; Wang, 2005; Wu et al., 2005), (2) high image quality schemes with a moderate embedding capacity (Chang, 2004; Wang et al., 2008; Wu and Tsai, 2003; Yang et al., 2008; Zhang and Wang, 2005), and (3) high embedding efficiency schemes with a slight distortion (Mielikainen, 2006; Zhang and Wang, 2006a, b). In the first type of schemes, the mechanism of capacity estimation in the embedding procedure functions on a pixel-by-pixel basis without taking the local texture into consideration. The most common embedding algorithm the first type has is the least significant bit (LSB) substitution technique, where the secret messages are hidden into the pixel LSB to create a stego-image. The second type is the adaptive steganographic schemes (Chang, 2004; Wang et al., 2008; Wu and Tsai, 2003; Yang et al., 2008; Zhang and Wang, 2005), where the embedding capacity estimation of a pixel depends on the variation among the immediate neighbor pixels. Finally, the third type is the high embedding efficiency schemes. They focus on how to minimize the image distortion when embedding relatively small amounts of messages, normally less than or equal to two bits per pixel. Zhang and Wang (2006b) introduced the embedding efficiency as the ratio between the number of embedded bits and the distortion energy caused by data embedding.

\section{PROPOSED METHOD}

For the method proposed in this study, the scheme chosen number two previously mentioned in the work is justified as a choice in the use of the sensitivity of the human visual system. Usually, this scheme is common to take the use of the frequency domain (Carvajal-Gámez, 2010). Within this domain can work discrete Fourier transform (DFT), discrete cosine transform (DCT) and the discrete wavelet transforms (DWT). Frequency domain methods hide messages in significant areas of the cover image which make them more robust to attacks, such as compression, cropping, and some image processing than the spatial domain methods. However, while they are robust to various kinds of signal processing attacks, they remain imperceptible to the human visual system (Johnson and Jajodia, 1998). The algorithm proposed here for the implementation of steganography is based on the DWT. In the optimization and evaluation of compression algorithms in digital images, the Peak Signal-to-Noise ratio (PSNR) is the most frequently used to evaluate the quality of the images (Huanga et al., 2005).

However, the use of qualitative measures of the image are based on the properties of HVS, then the models usually are embedded into HVS sensitivity to light and sensitivity to spatial frequency (Ginesu et al., 2006). If we apply the DWT, the image resolution is divided into 4 sub-matrices called approximations (a), horizontal $(h)$, vertical (v), and diagonal (d), each sub-matrix is a copy of the original one in different levels of the frequency which provides a certain amount of energy (Moon et al., 2007; Walker, 2003). In the subdivision of images, the steganography algorithm is applied only in the sub-matrix $h$ and the other sub-matrices are discarded. The proposed steganographic algorithm based on wavelets is shown in Figure 1 (Reddy and Chatterji, 2005). From the third block of Figure $1, \sigma_{R}$ (red channel, from RGB image) that defines the standard deviation of the cover image $x[n, m]$, with it we apply the following criterion of insertion:

$$
\sigma_{R} \leq x[n / 2, m / 2]
$$

then $y[n, m]$, where

$x[n, m]$ is the cover image and $y[n, m]$ is the hidden image (Figure 1).

DWT is closely linked to the analysis of multi-resolution (MRA), that is, see the signal at different frequencies (Vetterli and Kočević, 1995), which allows to have a broader knowledge of the signal and facilitates the rapid calculation when the wavelet family is orthogonal (Petrosian and Meyers, 2002; Debnath, 2002; 
Sheng, 2002; Bogges, 2009).

It can be obtained wavelets $\psi$ such that the family moved for $j$ and dilated for $n$,

$$
\left\{\psi_{j, n}(t)=\frac{1}{\sqrt{2^{j}}} \psi\left(\frac{t-2^{j} n}{2^{j}}\right)\right\}_{j, n \in Z^{2}}
$$

It is an orthonormal base of $L^{2}(I R)$. These orthogonal wavelets transport information about the changes of the signal to the resolution $2^{-j}$. Then, the MRA appears: an image will be modeled with orthogonal projections on vector space of different resolution, $P_{V_{j}} f, V_{j} \subset L^{2}(I R)$. The quantity of information in every projection will depend on the size of $\mathrm{V}_{\mathrm{j}}$. For search orthogonal wavelets it will be necessary to work with approaches of MRA (Petrosian and Meyers, 2002; Debnath, 2002; Sheng, 2002; Daubechies, 1988; Mallat, 1989). For a function $f \in L^{2}(I R)$, the partial sum of the coefficients wavelet $\sum_{n=-\infty}^{\infty}<f, \psi_{j, n}$ can be interpreted as the difference between two approaches of $f$ to the resolutions $2^{-1+j}$ and $2^{-j}$. The MRA approaches calculate the approach of signals to different resolutions with orthogonal projections in spaces $\left\{V_{j}\right\}_{j \in Z}$. Also, the MRA approaches are characterized completely by a particular discrete filter that controls the loss of information along the different resolutions. With these, discrete filters can be designed as orthogonal wavelets bases of simple form (Petrosian and Meyers, 2002; Debnath, 2002; Sheng, 2002; Daubechies, 1988; Mallat, 1989). The approach of a function

$f$ with a resolution $2^{-\mathrm{j}}$ comes specified by a discrete sampling grid, which provides local averages of $f$ in a neighborhood of proportional size to $2^{\mathrm{j}}$.

Therefore, a MRA approach consists of embedded networks approach. This means that the approach of a function to a resolution $2^{-j}$ is defined as an orthogonal projection in a space $\mathrm{V}_{\mathrm{j}} \subset \mathrm{L}^{2}(\mathrm{IR})$. The space $\mathrm{V}_{\mathrm{j}}$ regroups all the possible approaches to the resolution $2^{-j}$. The orthogonal projection of $f$ is the function $f_{j} \in V_{j}$ that it minimizes $\left\|f-f_{j}\right\|$. The orthonormal wavelets carry the necessary details to increase the resolution of the approach of the signal. The approaches of $f$ to the scales $2^{\mathrm{j}}$ and $2^{\mathrm{j}-1}$, are respectively equal, to its orthogonal projections in $\mathrm{V}_{\mathrm{j}}$ and $\mathrm{V}_{\mathrm{j}-1}$. We already know that $\mathrm{V}_{\mathrm{j}} \subset \mathrm{V}_{\mathrm{j}-1}$. Be $\mathrm{W}_{\mathrm{j}}$ the orthogonal complement of $\mathrm{V}_{\mathrm{j}}$ in $\mathrm{V}_{\mathrm{j}-1}$. The orthogonal projection of $f$ in $\mathrm{V}_{\mathrm{j}-1}$ can be written as the sum of orthogonal projections $P$ in $V_{j}$ and $W_{j}$. Then, $P_{V_{j-1}} f=P_{V_{j}}+P_{W_{j}}$. The function $f(t)$ can be reconstructed from the discrete wavelets coefficients $W_{f}(j, n)$ in the following way (Petrosian and Meyers, 2002; Debnath, 2002; Sheng, 2002; Daubechies, 1988; Mallat, 1989),

$$
f(t)=A \sum_{j n} \sum_{f}(j, n) \psi_{j, n}(t)
$$

Where $j$ is the scale factor and $n$ is the movement factor. The wavelets $\psi_{j n}(t)$ generated of the same wavelet mother function $\psi(t)$ have different scale $j$ and place $n$, but they have the same form. Scale factor $j>0$ is always used. The wavelet is dilated when the scale $j>1$, and it is contracted when $j<1$. This way, changing the value of $j$ the different range from frequencies is covered. Big values of the parameter $j$ correspond to frequencies of minor range, or a big scale of $\psi_{j, n}(t)$. Small values of $j$ correspond to frequencies of minor range or a very small scale of $\psi_{j, n}(t)$ (Sheng, 2002; Bogges, 2009; Petrosian and Meyers, 2002; Debnath, 2002; Sheng, 2002; Daubechies, 1988; Mallat, 1989). The continuous wavelet functions with discrete factors of scale and movement are named discrete wavelets. Finally, the signal $f(t)$ can be compressed or expand in the time. This will have a few certain after effects in the plane of frequencies,

$$
\begin{aligned}
& f(t) \text { compression by a factor } 2^{j}(s) f_{s}(t)=\frac{1}{\sqrt{2^{j}}} f\left(\frac{t}{s}\right) \\
& \hat{f}(w) \text { compression by a factor } \frac{1}{2^{j}} \hat{f}_{2^{j}}(w)=\frac{1}{\sqrt{2^{j}}} 2^{j} \hat{f}\left(2^{j} w\right)=\sqrt{2^{j}} \hat{f}\left(2^{j} w\right)
\end{aligned}
$$

The coefficient of the decomposition of a function $f$ in an orthogonal base of wavelets is calculated by a subsequent algorithm of discrete convolution with $h$ and $g$, and realizes a sampling as follows,

$\mathrm{x}_{\mathrm{low}}[\mathrm{k}]=\sum_{\mathrm{n}} \mathrm{x}[\mathrm{n}] \mathrm{h}[2 \mathrm{k}-\mathrm{n}]$

$\mathrm{X}_{\text {high }}[\mathrm{k}]=\sum_{\mathrm{n}} \mathrm{X}[\mathrm{n}] \mathrm{g}[2 \mathrm{k}-\mathrm{n}]$

Where $x_{\text {low }}[\mathrm{k}]$ and $x_{\text {high }}[\mathrm{k}]$ are outputs of the low pass filter (LPF) and high pass filter (HPF), respectively; $\mathrm{g}[2 \mathrm{k}-\mathrm{n}]$ and $\mathrm{h}[2 \mathrm{k}-\mathrm{n}]$ represent the impulse response of HPF and LPF, respectively, subsampled by a factor of 2 as expressed in Equations 4 and 5 (Walker, 2003; Vetterli and Kočević, 1995; Daubechies, 1988; Mallat, 1989).

These coefficients are calculated by cascades of discrete filters through convolution and sampling.

Then the host image $x[n, m]$ must pass through a series of mirror filters banks in quadrature (Moon et al., 2007; Vetterli and Kočević, 1995; Walker, 2003), these filters are LPF and HPF. The signal from each filter is decimated by a factor of 2 . The filter $h$ removes de high frequencies of the $x[n, m]$, while $g$ removes the lower frequencies. For the reconstruction, an interpolation is realized, inserting zeros and expanding $x_{\text {high,high, }} x_{\text {high,low, }} x_{\text {low,high, }} x_{\text {low,low. }}$ This filtering and decimation process in a continuous way is known as sub-band coding. The Figure 2 depicts the decomposition of the discrete wavelet for an RGB image and is interpreted as the decomposition of the sub-matrix $R$ represented by $X_{R}[n, m]$, for the first decomposition shown in Figure 2 which applies to itself step of the LPF through the rows and columns to obtain sub-matrix a previously mentioned of the image, in the second decomposition is applied to LPF by filtering the rows and columns is applied to HPF obtaining the sub-matrix $h$, the third decomposition is similar to $h$ but reverses the first filter is the HPF and later the LPF and get the sub-matrix $v$ and finally for sub-matrix $d$ filtering is applied in rows and columns with the HPF. This operation is interpreted as follows (Vetterli and Kočević, 1995; Walker, 2003; Daubechies, 1988; Mallat, 1989).

The DWT decomposes a discrete signal into two sub-signals of 

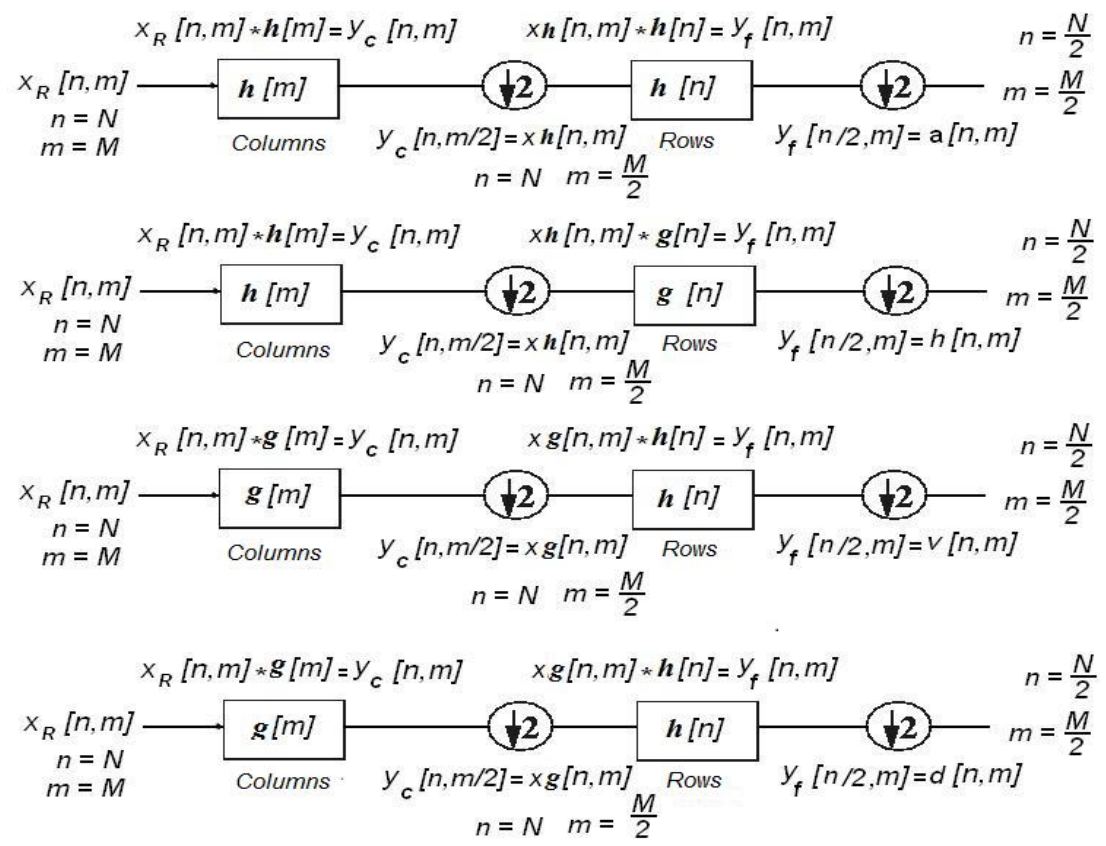

Figure 2. Filters bank for encoding sub-bands where it represents discrete wavelet decomposition of an image.

half of the original length. This sub-signal is known as the approaches and the other one is known as the details (Walker, 2003). The first sub-signal $a^{1}=\left(a_{1}, a_{2}, \cdots, a_{m / 2}\right)$, for the signal $x$ is obtained making the average of the signal as follows: the first value $a_{1}$ is calculated by taking the first set of values vector $x[m]:\left(x_{1}+x_{2}\right) / 2$ and multiplying it by $\sqrt{2}$, that is, $a_{1}=\left(x_{1}+x_{2}\right) / \sqrt{2}$, similarly $a_{2}=\left(x_{3}+x_{4}\right) / \sqrt{2}$, etc. It is given by Walker (2003) as follows:

$a_{m / 2}=\frac{x_{2 m-1}+x_{2 m}}{\sqrt{2}}$

Where $m$ is the total number of vectorial elements. The other subsignal is also known as the first fluctuation of the signal $x$ denoted as: $d^{1}=\left(d_{1}, d_{2}, \cdots, d_{m / 2}\right)$ and is calculated by taking the difference between the first pair of values of $x,\left(x_{1}-x_{2}\right) / 2$ and then multiplied and divided by $\sqrt{2}$, and so on. The expression can be written in the following way,

$d_{m / 2}=\left(\frac{x_{2 m-1}-x_{2 m}}{\sqrt{2}}\right)$

Where $m$ is the vector size. After applying, the DWT got two vectors, which are approximations and details obtained, with a length of half the original vector. Finally, continuing the recovery of the vector,

$$
f[n]=\left\{\frac{a_{1}+d_{1}}{\sqrt{2}}, \frac{a_{1}-d_{1}}{\sqrt{2}}, \cdots, \frac{a_{n / 2}+d_{n / 2}}{\sqrt{2}}, \frac{a_{n / 2}-d_{n / 2}}{\sqrt{2}}\right\}
$$

We note that the terms $a_{1}$ and $d_{1}$ in Equations 6 and 7 can be interpreted as follows

$$
\begin{aligned}
& \varepsilon_{\left(a^{1} \mid d^{1}\right)}=a_{1}+\cdots+a_{n / 2}+d_{1}+\cdots+d_{n / 2}, \\
& a_{1}+d_{1}=\left[\frac{f_{1}+f_{2}}{\sqrt{2}}\right]^{2}+\left[\frac{f_{1}-f_{2}}{\sqrt{2}}\right]^{2}=\frac{f_{1}^{2}+2 f_{1} f_{2}+f_{2}^{2}}{2}+\frac{f_{1}^{2}-2 f_{1} f_{2}+f_{2}^{2}}{2}=f_{1}^{2}+f_{2}^{2}
\end{aligned}
$$

and similarly for each set of vectorial approaches and details (Walker, 2003). So, the conservation of energy in wavelets is mentioned, the factor $1 / \sqrt{2}$ is mentioned too (Carvajal-Gámez, 2010). By applying the steganographic algorithm to the $h$ submatrix, it is necessary to use the scaling factor, but as the work is with an 8-bit RGB image, this scaling factor is closely related to energy conservation applied to wavelet theory for grayscale images as shown in most applications. However, for RGB images we propose the following scaling factor,

$1 / \sqrt{2^{j}}$

Where $j$ is directly dependent on the number of bits that integrate the image (Carvajal-Gámez, 2010).

\section{EXPERIMENTAL RESULTS}

Many distortion or quality measures used in the images during the visual information processing belong to the group of measures of difference in distortion (Kutter and Petitcolas et al., 1999; Carvajal-Gámez, 2010), which are based on the difference between the original and 
modified images. The most common distortion measure is the peak signal to noise relation PSNR,

$\operatorname{PSNR}=10 \cdot \log \left[\frac{(255)^{2}}{\mathrm{MSE}}\right], \mathrm{dB}$

Where $\quad M S E=\frac{1}{M_{1} M_{2}} \sum_{n=1 m=1}^{M_{1} M_{2}}\|y(n, m)-x(n, m)\|_{L_{2}}^{2} \quad$ is the mean square error, $M_{1}, M_{2}$ are the image dimensions, $y(n, m)$ is the $3 \mathrm{D}$ vector value of the pixel $(n, m)$ of the stego-image, $x(n, m)$ is the corresponding pixel in the original cover image, and $\|\cdot\|_{L_{1}},\|\cdot\|_{L_{2}}$ are the L1- and L2-vector norms, respectively; The normalized color deviation (NCD) is used for the quantification of the color perceptual error,

$$
N C D=\frac{\sum_{n=1}^{M_{1}} \sum_{m=1}^{M_{2}}\left\|\Delta E_{L u v}(n, m)\right\|_{L_{2}}}{\sum_{n=1}^{M_{1}} \sum_{m=1}^{M_{2}}\left\|E_{L u v}^{*}(n, m)\right\|_{L_{2}}}
$$

Here, $\left\|\Delta E_{L u v}(n, m)\right\|_{L_{2}}=\left(\Delta L^{*}(n, m)\right)^{2}+\left(\Delta u^{*}\right)^{2}+\left(\Delta v^{*}\right)^{2}$ is the norm of color error; $\Delta L^{*}, \Delta u^{*}$, and $\Delta v^{*}$ are the difference in the $L^{*}, u^{*}$, and $v^{*}$ components, respectively, between the two color vectors that present the stego and cover images for each a pixel $(n, m)$ of an image, and $\left\|E_{L u v}^{*}(i, j)\right\|_{L_{2}}=\left[\left(L^{*}\right)^{2}+\left(u^{*}\right)^{2}+\left(v^{*}\right)^{2}\right]^{1 / 2}$ is the norm or magnitude of the host image pixel vector in the $L^{*} u^{*} v^{*}$ space.

The quality index $(Q)$ is provided to demonstrate the quality of the stego-images (Carvajal-Gámez, 2010),

$$
\mathrm{Q}=\frac{4 \sigma_{x y} x y}{\left(\sigma_{x}^{2}+\sigma_{y}^{2}\right)\left(x^{2}+y^{2}\right)}
$$

Where $x$ and $y$ are the mean values of host and stegoimages, respectively, $\sigma_{x}^{2}$ and $\sigma_{y}^{2}$ are the variances of host and stego-images, respectively, and $\sigma_{x y}=\frac{1}{N-1} \sum_{i}^{N}\left(x_{i}-x\right)\left(y_{i}-y\right)$.

Some tests were conducted with 8-bit RGB images to show our mentioned scaling factor in this paper. As mentioned previously, a filter can distort the images, as will be shown in subsequent tests applied filters to distort the host image with the DWT. However, applying the proposed scaling scheme can be seen as an improvement in visual images. The proposed scheme has been evaluated, and its performance is compared with the LSB (Chan and Cheng, 2004), LSB optimized (LSBO) (Yuan et al., 2007) steganographic methods. These methods were implemented to compare them with the proposed scheme approach. The reason for selecting these methods of comparison is that their performances have been compared with various well known methods, and they were used accordingly as the reference ones. From these results we present a discussion in terms of the objective image quality, the $\mathrm{HC}$, the color spaces, and the subjective visual results, and we provide recommendations to use the proposed methods under different circumstances. Table 1 shows the performance results in terms of PSNR, mean absolute error (MAE), $\mathrm{COI}$ (Correlation), Q, NCD, and HC. In the case of different $j$ values in the scaling factor by using the $320 \times$ 320 color images "Mandrill" (Vitterbi, 2012) as cover image and "Lena" (Vitterbi, 2012) as hide image, from Table 1, one can see when the $j$ value increases the performance results increase too. Figure 3 depicts the processed images for stego-image Mandrill (Figure 3a, b, and c) and retrieved secret image Lena (Figure $3 d$, e, and f) according with Table 1 . Table 1 shows that as the value of $j$ is increased, measures considerably improve visual quality. It can be seen that when $j=0$, the values of stego-imagen "Mandrill" in PSNR is equal to $31.5084 \% \mathrm{db}$ with a COI equal to $78.51 \%$, similarly to the recovered image "Lena" which is a test that more affected by the distortion has a PSNR equal to $16.5327 \mathrm{db}$ and a $\mathrm{COI}$ equal to $38.8 \%$. Also, one can observe that when $j$ is increased to 5, it obtained PSNR value of $36.1233 \mathrm{db}$ for stego-image and the recovered image with a PSNR of $27.2474 \mathrm{db}$. We observe from Figure $3 \mathrm{c}$ and $f$ that the best results are obtained when $j=9$, where $j$ represents the number of bits resolution of the image to hide. It is observed that when $j$ is gradually increased, the quality image is significantly improved. From Figure $3 d$, e, and $f$ one can see that when the value of proposed scaling factor increases the subjective quality of images increases too, it is observed that Figures $3 a, b$, and $c$ have in the upper part a certain lineal distortion, which can be interpreted as external information inserted to the cover image. As $j$ takes the value of 10 or 24 of this lineal distortion in the top of the stego-image not easily identified visually. We also present the wrong images. Table 2 shows the results using comparative methods, LSB, LSBO and WLSB.

Test were performed with $j=10$, in Table 1 and shows the performance results in the case of use in the scaling factor. More tests were performed to increase the value of $j$ to $j=24$, amount determined by the image type manipulated. The significant changes of stego-image and the recovered image were observed. Figure 4 presents the visual results according to Table 3 . The proposed scaling factor $1 / \sqrt{2^{j}}$ for each test presents a different result as can be seen in the previous tests, the scaling 
Table 1. Performance results for different values of $j$ with cover image "Mandrill" and hidden image "Lena".

\begin{tabular}{llccccc}
\hline & $\boldsymbol{j}$ & PSNR db & COI \% & NCD & Q & MAE \\
\hline \multirow{2}{*}{$j=0$} & Cover image & 31.5084 & 78.52 & - & 0.7836 & 10.4878 \\
& Recovered image & 16.5327 & 38.80 & 0.4748 & 0.3586 & 4.9403 \\
& & & & & & \\
$j=2$ & Cover image & 31.4999 & 80.46 & - & 0.8040 & 9.9490 \\
& Recovered image & 16.9537 & 38.89 & 0.4749 & 0.3587 & 4.9471 \\
& Cover image & 36.1233 & 97.81 & 0.0020 & 0.9792 & 3.2086 \\
$j=5$ & Recovered image & 27.2474 & 99.85 & 0.0020 & 0.9962 & 2.7714 \\
& Cover image & 36.1233 & 99.08 & $8.015 \mathrm{e}-4$ & 0.9913 & 2.0309 \\
$j=9$ & Recovered image & 31.0781 & 99.80 & 0.0020 & 0.9962 & 2.7714 \\
& & & & & & \\
\multirow{3}{*}{$j=10$} & Cover image & 36.1233 & 99.34 & $6.0486 \mathrm{e}-4$ & 0.9934 & 1.7022 \\
& Recovered image & 32.5167 & 99.55 & 0.0020 & 0.9962 & 2.7714 \\
\hline
\end{tabular}

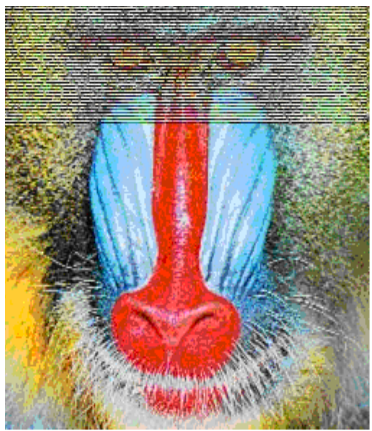

a)

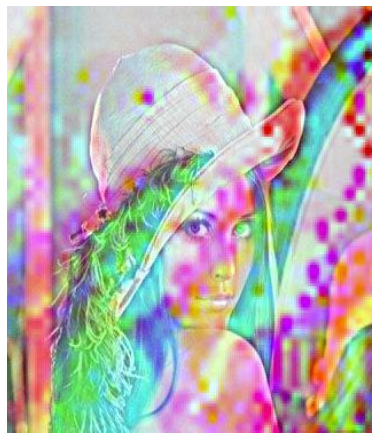

d)

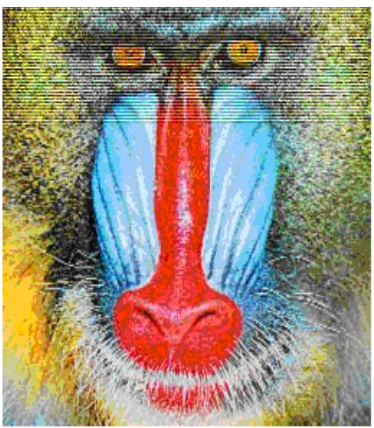

b)

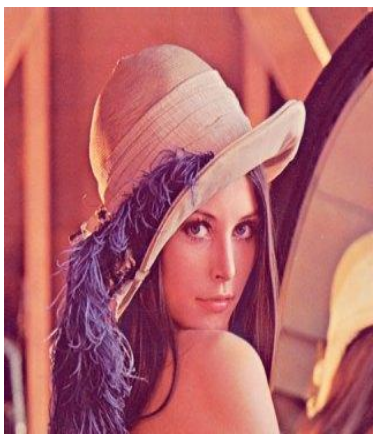

e)

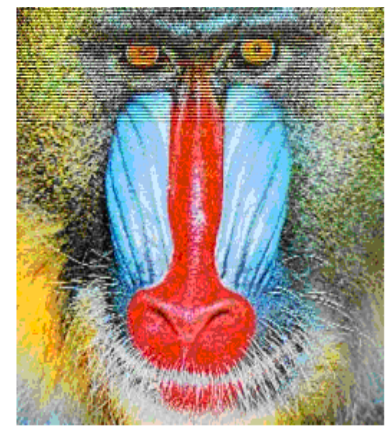

c)

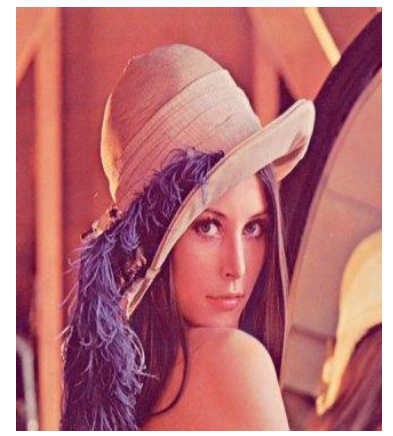

f)

Figure 3. Visual results for different values of $j$ in the scaling factor, a) and d) column with $j$ $=2$, b) and e) column with $j=5$, and c) and f) $j=9$.

factor does not affect the steganographic algorithm and preserving the energy of images. It can be seen in the Lena error image of Figure $4 d$, the difference in values between the host image and the recovered image is approximately zero. In general, we can say that in the case of the cover images with uniform regions (Table 4, 'Splash' image as cover image, Figure 5), although conserved, acceptable results quality image, compared with LSB, LSBO and WLSB methods there is no significant difference of values. Significantly for uniform color images insertion is usually more complicated because of the few regions of abrupt changes. In the opposite case, the best scenario is when the cover images contain several details (Table 3, 'Mandrill' image as cover image) where we can observe that the PSNR, $\mathrm{COI}, \mathrm{NCD}, \mathrm{MAE}$ and $\mathrm{Q}$ of the proposed method is higher than other methods used as comparative providing the best image quality in favor of the proposed methods. Then, the hidden information is almost imperceptible. The results shown in Table 3 using the scaling factor $j=24$ 
Table 2. Performance results for different steganographic methods with cover image "Mandrill" and hidden image "Lena".

\begin{tabular}{llccccc}
\hline \multicolumn{2}{c}{ Parameter } & PSNR db & COI \% & NCD & Q & MAE \\
\hline \multirow{2}{*}{ LSB } & Cover image & 34.5602 & 99.78 & $7.3506 \mathrm{e}-4$ & 0.9977 & 3.5758 \\
& Recovered image & 36.1233 & 99.54 & 0.0020 & 0.9962 & 2.7714 \\
& & & & & & \\
\multirow{2}{*}{ LSBO } & Cover image & 26.9908 & 99.76 & 0.0022 & 0.9948 & 10.6396 \\
& Recovered image & 29.2421 & 99.54 & 0.0033 & 0.9944 & 8.0316 \\
& & & & & & \\
\multirow{2}{*}{ WLSB } & Cover image & 28.8347 & 99.77 & 0.0053 & 0.9963 & 8.8469 \\
& Recovered image & 31.2219 & 99.54 & 0.0033 & 0.9954 & 5.0316 \\
\hline
\end{tabular}

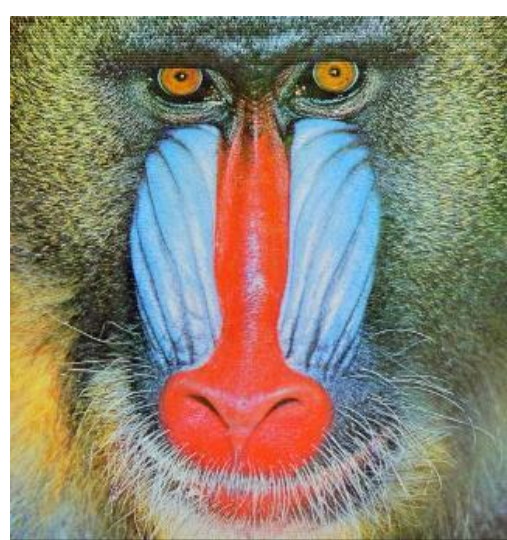

a)

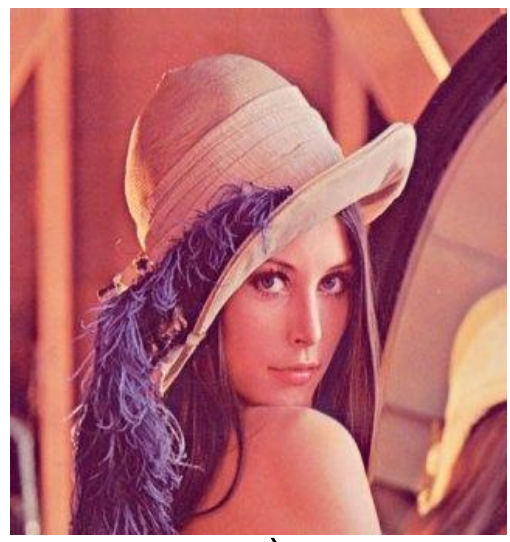

c)

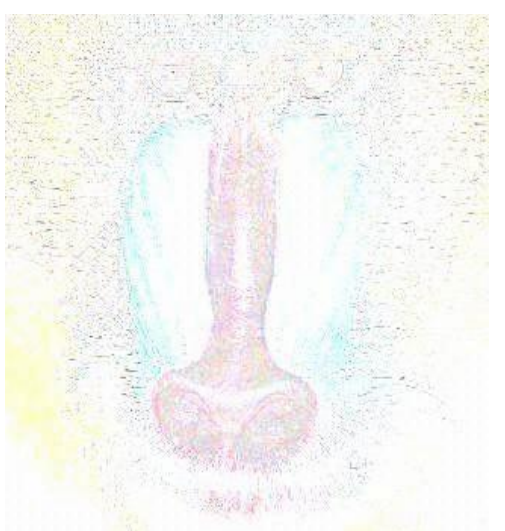

b)

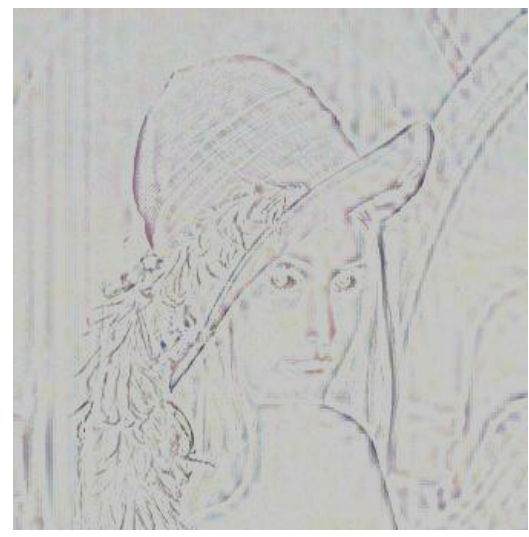

d)

Figure 4. Visual results in the case of $j=10$, a) host image "Mandrill, b) error image "Mandrill, c) hide image "Lena", d) error image "Lena".

are superior to those shown in Table 2. Being the best method, LSB with PSNR equal to $34.5602 \mathrm{db}$ is exceeded by the second PSNR of $36.7973 \mathrm{db}$ with $j=24$. Tables 4 and 5 show the results for the stego-image "Splash" (image to hide "Tiffany") and the recovered image "Tiffany", respectively. Figure 5 presents the results obtained from subjective visual Tables 4 and 5 .

\section{Conclusions}

The RGB images are altered in their energy contribution in each sub-matrix of wavelet decomposition when the steganographic algorithm is applied. It is known that the value of $1 / \sqrt{2}$ is the key factor in the adjustment of the wavelets energy; this adjustment value has been applied 
Table 3. Performance results in the case of $j=24$ in the scaling factor with cover image "Mandrill" and hidden image "Lena".

\begin{tabular}{ll}
\hline Cover image "Mandrill" & Hide image "Lena" \\
\hline $\mathrm{Q}=0.9968$ & $\mathrm{Q}=0.9962$ \\
$\mathrm{PSNR}=36.7973 \mathrm{~dB}$ & $\mathrm{PSNR}=36.1232 \mathrm{~dB}$ \\
$\mathrm{COI}=99.67 \%$ & $\mathrm{COI}=99.56 \%$ \\
$\mathrm{NCD}=5.7204 \mathrm{e}-4$ & $\mathrm{NCD}=19.735 \mathrm{e}-4$ \\
$\mathrm{MAE}=1.4721$ & $\mathrm{MAE}=2.7714$ \\
\hline
\end{tabular}

Table 4. Performance results for different steganographic methods with cover image "Splash" and hidden image "Tiffany".

\begin{tabular}{lcccc}
\hline \multirow{2}{*}{ Test } & \multicolumn{4}{c}{ Steganographic algorithms } \\
\cline { 2 - 5 } & LSB & LSBO & WLSB & Proposed method $\boldsymbol{j}=\mathbf{2 4}$ \\
\hline PSNR dB & 30.1606 & 25.8026 & 26.0296 & 34.5830 \\
MAE & 6.1696 & 11.3249 & 11.4953 & 3.5761 \\
COI \% & 99.10 & 99.08 & 99.18 & 99.60 \\
Q & 0.9871 & 0.9828 & 0.9881 & 0.9919 \\
NCD & 0.0065 & 0.0101 & 0.0086 & 0.0028 \\
\hline
\end{tabular}

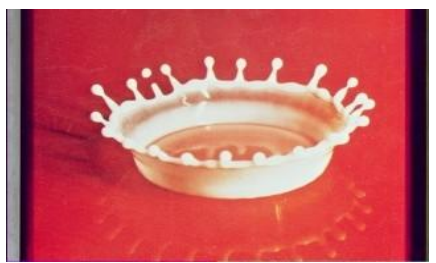

a)

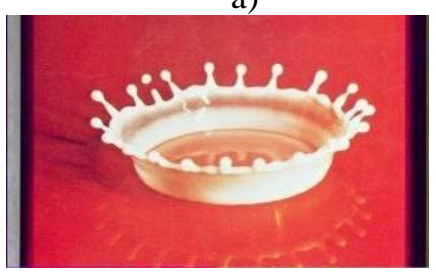

c)

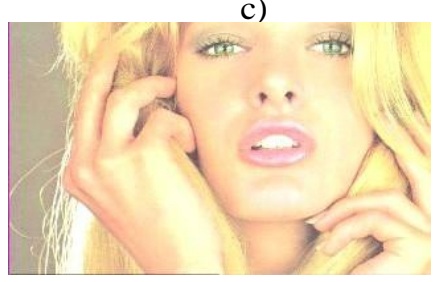

e)

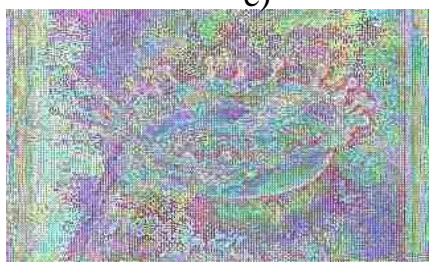

g)

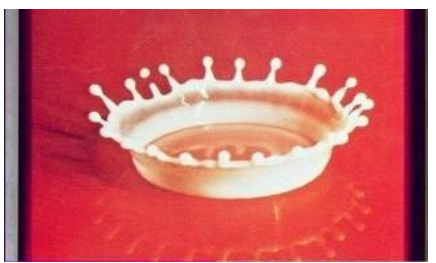

b)

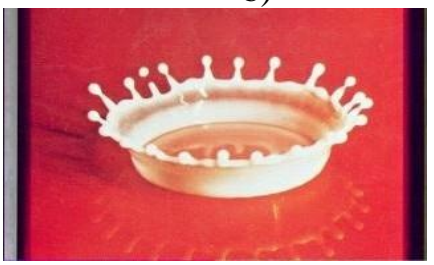

d)

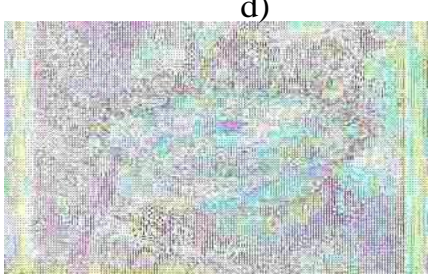

f)

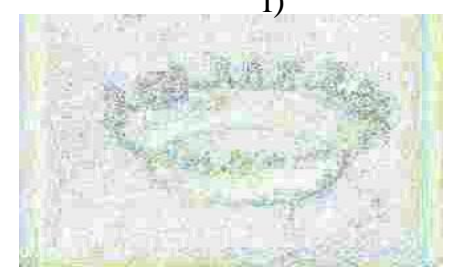

h)

Figure 5. a) Cover image "Splash", Stego-images; b) LSB; c) LSBO; d) proposed method with $\mathrm{j}=24$; e) Image Original "Tiffany", error stego-images; f), 4LSB; g), 4LSBO; h), proposed method. 
Table 5. Comparative performances of the recovered image "Tiffany".

\begin{tabular}{lcccc}
\hline \multirow{2}{*}{ Test } & \multicolumn{4}{c}{ Steganographic algorithms } \\
\cline { 2 - 5 } & LSB & LSBO & WLSB & Proposed method $\mathbf{j}=\mathbf{2 4}$ \\
\hline PSNR dB & 34.3755 & 29.7652 & 32.2852 & 34.3755 \\
MAE & 3.4600 & 6.9246 & 4.4938 & 3.4600 \\
COI \% & 98.37 & 98.06 & 97.99 & 98.37 \\
Q & 0.9713 & 0.9650 & 0.9788 & 0.9713 \\
NCD & 0.00065 & 0.0012 & 0.0028 & 0.00065 \\
\hline
\end{tabular}

only in gray scale images tests. However, the energy conservation factor mentioned above is not valid for true color images, such as the one formed by 3 sub-matrices (RGB) which separately provides some level of energy, must have a value close to the total energy calculated up to the image, because if it is not right or there are energy gap in the components, the images may have a poor display as distortion. By applying the proposed scaling factor, $1 / \sqrt{2^{j}}$, where $j$ represents the number of bits that integrates each image, there is an adjustment factor for the energy input in each sub-matrix. It is also noted that when it is changing the value of $j$, adjust the sharpness and image clarity providing a visible improvement of the visual image. The results shown here with varying scale factor were showing improvement in each of the visual quality measures, comparing $j=10$ exceeded the values obtained as the LSB, LSBO and WLSB steganographic methods. Agree with the experimental results we observed that there are some variations in the PSNR, $M A E, N C D, C O I$ and $Q$ results between different versions of the steganography methods but in general, we recommend the application of scaling factor because they guarantee good results in comparison with other methods used as comparative, and sometimes these provide a higher performance than other variants of the steganography methods. By other way, the scaling factor, provide the best results in almost all the tests by balancing the performance of the image quality among different types of images (with uniform regions or several details).

\section{ACKNOWLEDGMENTS}

This work is supported by National Polytechnic Institute of Mexico and National Council on Science and Technology (CONACYT) Mexico.

\section{REFERENCES}

Bogges A, Narcovich F (2009). A first course in wavelets with Fourier Analysis, 2th Ed., Wiley pp. 1-305.

Carvajal-Gámez BE, Gallegos Funes FJ, Lopez Bonilla JL (2010). Computational Techniques and algorithms for Image Processing. Ed. Lambert Academic Publishing Chapter 13.
Chan C, Cheng (2004). Hiding data in images by simple LSB substitution. Pattern Recogn. 37:469-474.

Cheddad A, Condell J, Curran K, Mc Kevitt P (2010). Digital image steganography: Survey and analysis of current methods. Signal Process. 90:727-752.

Daubechies I (1988). Orthornormal bases of compactly supported wavelets. Commun. Pure Appl. Math. 41:909-996.

Debnath L (2002). Wavelets and Signal Processing. Birkhauser, Berlin P. 106.

Ginesu G, Massidda F, Giusto DD (2006). A multi-factors approach for image quality assessment based on a human visual system model. Signal Process. Image Commun. pp. 316-333.

Huanga KQ, Wub ZY, Fungc GSK, Chan HY (2005). Color image denoising with wavelet thresholding based on human visual system model. Signal Process. Image Commun. pp. 115-127.

Johnson NF, Jajodia S (1998). Exploring Steganography: Seeing the Unseen. Comput. Pract. IEEE pp. 26-34.

Johnson NF, Jajodia S (1998). Steganalysis of Images Created Using Current Steganography Software. Lecture notes in computer science. Springer Verlag (1525):273-289.

Kutter M, Petitcolas F (1999). A fair benchmark for image watermarking systems. Electronic Imaging '99. Security and Watermarking of Multimedia Contents. Int. Soc. Opt. Eng. 3657:1-14.

Lin C, Tsai W (2004). Secret image sharing with steganography and authentication. J. Syst. Softw. 73:405-414.

Mallat S (1989). A theory for multiresolution signal decomposition: the wavelet representation. IEEE Trans. Pattern Anal. Mach. Intell. 11(7):674-694.

Mielikainen J (2006). LSB matching revisited. IEEE Signal Process. Lett. 13:285-287.

Moon H, You T, Sohn M, Kim H, Jang D (2007). Expert system for low frequency adaptive image watermarking: Using psychological experiments on human image perception. Expert Syst. Appl. pp. 674686.

Petitcolas F, Anderson RJ, Kuhn MG (1999). Information hiding a survey. Proceed. IEEE 87(7):1062-1078.

Petrosian A, Meyers F (2001). Wavelets in signal and image analysis. Comput. imaging vision Springer. P. 19.

Petticolas F, Katzenbeisser S (2000). Information hiding techniques for steganography and digital watermarking. Artech. House. Boston. pp. 15-30.

Reddy A, Chatterji B (2005). A new wavelet based logo-watermarking scheme. Pattern Recog. Lett. pp. 1019-1027.

Sheng $Y$ (2002). The transforms and applications handbook. Ed. 2th, CRC Press EEUU.

Thien C, Lin J (2003). A simple and high-hiding capacity method for hiding digit by digit data in images based on modulus function. Pattern Recog. 36:2875-2881.

Vetterli M, Kočević J (1995). Wavelets and subband coding. PrenticeHall, New Jersey. pp. 1-9, 97-202, 209-298, 414-438.

VITTERBI (2012). Data Base Images of the University of Southern of California, Signal and image processing institute. http://sipi.usc.edu/database.

Walker J (2003). A primer on wavelets and their scientific applications, Chapman and Hall/CRC, London. pp. 5-26, 41-53.

Wang C, Wu N, Tsai C, Hwang M (2008). A high quality steganographic 
method with pixel value differencing and modulus function. J. Syst. Softw. 81:50-58.

Wang J (2005). Steganography of capacity required using modulo operator for embedding secret image. Appl. Math. Comput. 164:99116.

Wu D, Tsai W (2003). A steganographic method for images by pixel value differencing. Pattern Recogn. Lett. 24:1613-1626.

Wu H, Wu N, Tsai C, Hwang M (2005). Image steganographic scheme based on pixel-value differencing and LSB replacement methods. IEEE Proceed. Vision Image Signal Process. 152:611-615.

Wu N, Hwang M (2007). Data hiding: Current status and key issues. Int. J. Network Secur. 4:1-9.

Yang C, Weng C, Wang S, Sun H (2008). Adaptive data hiding in edge areas of images with spatial LSB domain systems. IEEE Trans. Inform. Forensics Secur. 3:488-497.
Yuan HY, Chin CC, luon CL (2007). A new steganographic method for color and grayscale image hiding. Comput. Vision Image Unders. 107:183-194.

Zhang X, Wang S (2005). Steganography using multiple-base notational system and human vision sensitivity. IEEE Signal Process. Lett. 12:67-70.

Zhang X, Wang S (2006a). Dynamical running coding in digital steganography. IEEE Signal Process. Lett. 13:165-168.

Zhang X, Wang S (2006b). Efficient steganographic embedding by exploiting modification direction. IEEE Commun. Lett. 10(11):781783. 